\title{
Two operative cases of abdominal aortic aneurysm with isolated dissection of the abdominal aorta
}

\author{
Naoki Takeno ${ }^{1}$, Yasuyuki Shimada ${ }^{2}$, and Yasushi Terada ${ }^{2}$ \\ ${ }^{1}$ Department of General Medicine, Shonai-Amarume Hospital, Japan \\ ${ }^{2}$ Department of Cardiovascular Surgery, Shonai-Amarume Hospital, Japan
}

\begin{abstract}
Objective: Isolated abdominal aortic dissection (IAAD) co-occurring with an abdominal aortic aneurysm (AAA) is rather rare. The objective of this report was to discuss the adequate timing and method of surgery for this condition.

Patients: We encountered two operative cases, for which we carefully considered the timing and method of surgery. One patient underwent open repair 1 month after the onset, and the other patient underwent endovascular aneurysm repair (EVAR) 3 years after the onset.

Results: Both patients had a good postoperative recovery and are doing well 8 months after the surgery.

Conclusion: The presence of symptoms or an increase in the diameter of an AAA is important in determining the timing of intervention.
\end{abstract}

Key words: isolated abdominal aortic dissection, abdominal aortic aneurysm, penetrating atherosclerotic ulcers, surgery

\section{Case 1}

A 72-year-old-man visited our hospital 3 years ago because of a sudden onset of abdominal pain. He was diagnosed with isolated abdominal aortic dissection (IAAD) associated with an infrarenal saccular abdominal aortic aneurysm (AAA) (Figures 1 and 2). The diameter of the aneurysm was $25 \mathrm{~mm}$. Because the pain was transient, we performed follow-up with the patient under medical treatment, and checked the size of the AAA every 3 months with computed tomography (CT) or ultrasound echography. Three years after the onset, when he was 75 years old, he experienced abdominal discomfort and visited our hospital. The location of the dissection was unchanged; however, the diameter of the aneurysm on CT increased to $31 \mathrm{~mm}$ (Figures 1-3). We chose endovascular treatment (endovascular

Received: September 10, 2020

Accepted: December 14, 2020

Correspondence: Naoki Takeno, Department of Cardiovascular Surgery, Shonai-Amarume Hospital, 1-1-1 Shoyo, Shonai, HigashiTagawa, Yamagata 999-7782, Japan

E-mail: t.naoki8428@gmail.com

This is an open-access article distributed under the terms of the Creative Commons Attribution Non-Commercial No Derivatives (by-nc-nd) License <http://creativecommons.org/ licenses/by-nc-nd/4.0/>. aneurysm repair [EVAR]), and successfully performed the procedure. He is doing well 8 months after EVAR.

\section{Case 2}

A 71-year-old-man experienced severe low back pain that did not disappear for 2 weeks. Enhanced thin-slice CT scan showed a $>2 \mathrm{~cm}$ local dissection of the abdominal aortic wall (Figure 4). The CT scan also showed a saccular AAA with a diameter of $24.5 \mathrm{~mm}$ (Figures 5 and 6). We selected open surgery. We did not choose EVAR because we were concerned about the risk of rupture during catheter manipulation, as the wall of the aneurysm may be fragile in the acute phase of dissection. During surgery, we confirmed that the wall of the aneurysm was very thin (Figure 7). The postoperative course was uneventful, and he is doing well 8 months after the surgery.

\section{Informed consent and ethical considerations}

This study was conducted in accordance with the Declaration of Helsinki.

\section{Discussion}

IAAD is rare disease entity accounting for $1-4 \%$ of all aortic dissections ${ }^{1}$. Studies have shown that in more than 


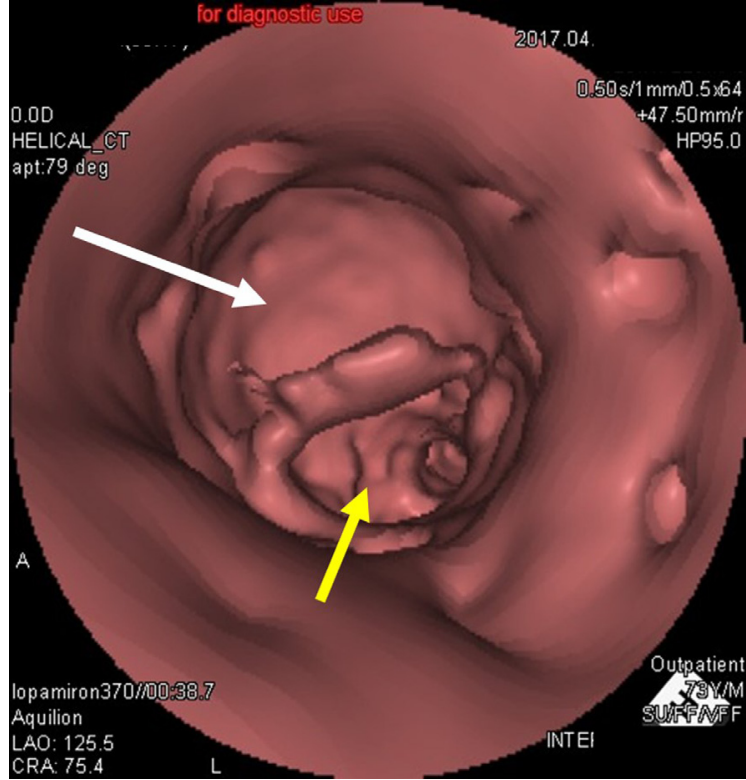

Figure 1 CT scan (intra-aortic view) of case 1. Local dissection of the abdominal aorta (entrance of the saccular aneurysm) from the proximal site is shown. The white arrow points to the entrance of the saccular aneurysm, which is occupying the upper half of the aorta. Bifurcation of common iliac arteries (yellow arrow) through the true lumen can be seen.

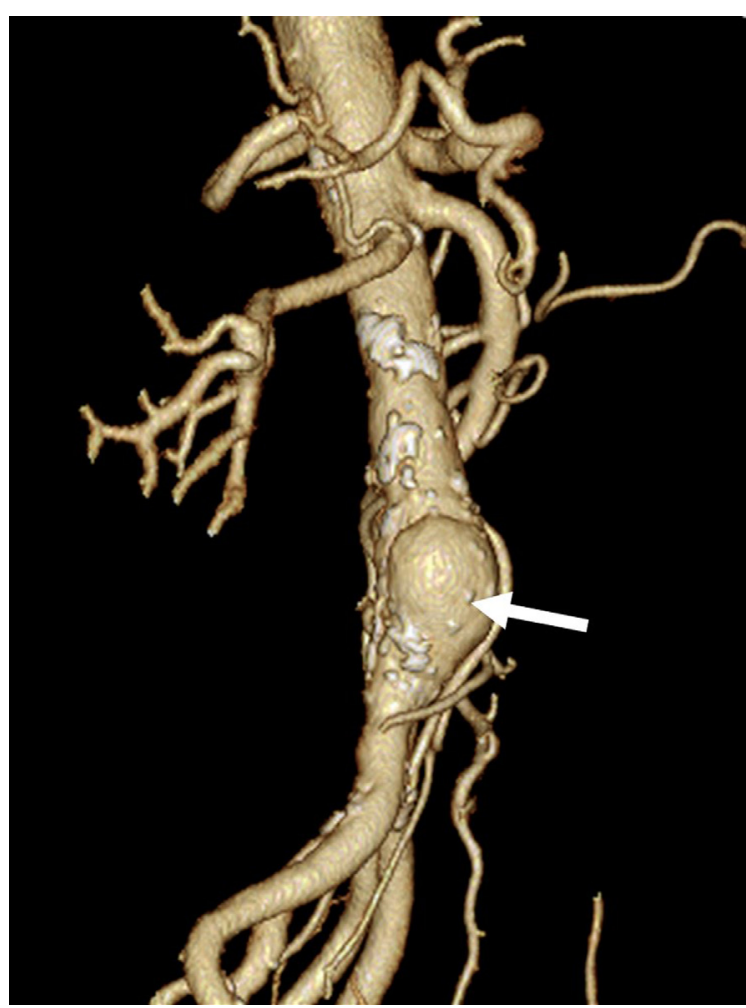

Figure 2 Enhanced three-dimensional CT scan of case 1. The white arrow points to the saccular aneurysm.

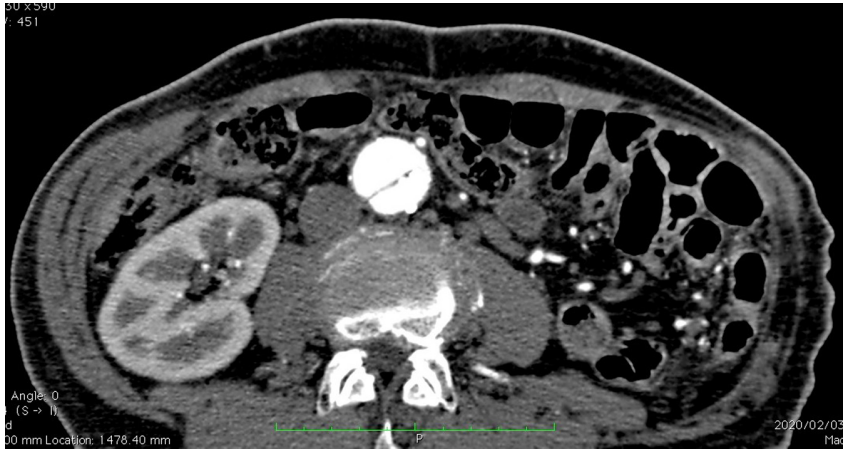

Figure 3 Enhanced CT scan of case 1 showing dissection in the abdominal aorta and a patent false lumen on the ventral side.

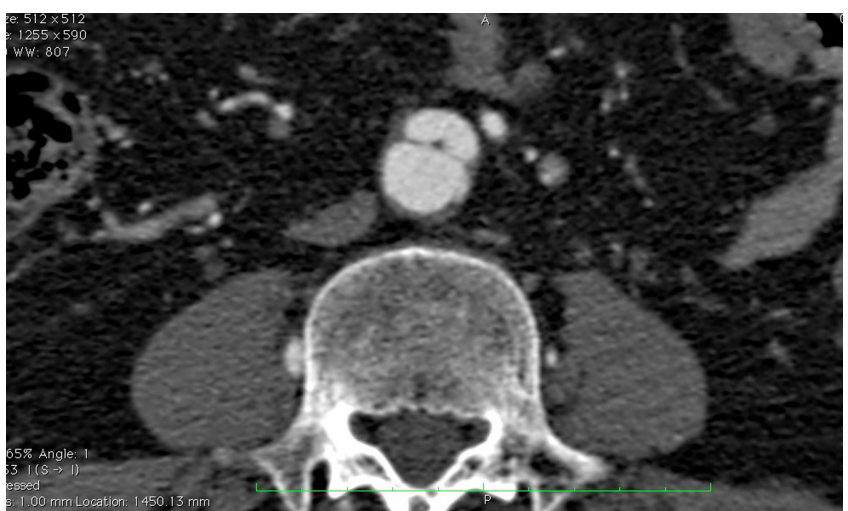

Figure 4 Enhanced CT scan of case 2 showing a $>2 \mathrm{~cm}$ dissection in the aortic wall.

half of the cases, IAAD leads to AAA development ${ }^{1,2)}$. Some review articles strongly suggested that IAAD is a complication of penetrating atherosclerotic ulcers (PAUs), ${ }^{3}$. In our two cases, the intra-aortic view of three-dimensional CT (enhanced medium is not required but thin-slice imaging is necessary) clearly showed a punched-out appearance of the entrance of the AAA in the aortic wall (Figures 1 and 5 ). The usefulness of intra-aortic imaging has recently been emphasized for transcatheter aortic valve implantation ${ }^{5}$. Enhanced CT angiography is an absolute requirement, as in type B aortic dissection. These imaging modalities allow precisely diagnosing the location of the tear in the intima and the shape of the aneurysm. We believe that PAUs develop into an IAAD and that IAAD induces saccular AAA development.

No established guidelines exist for the treatment of AAA with IAAD because of the limited number of cases. Most reports describe that the initial symptom is pain. Persistent pain indicates that dissection of the aortic wall is progressing and that immediate therapeutic intervention is necessary ${ }^{2,6)}$, as in type B aortic dissection with or without AAA. When IAAD involves an AAA but the pain is under 


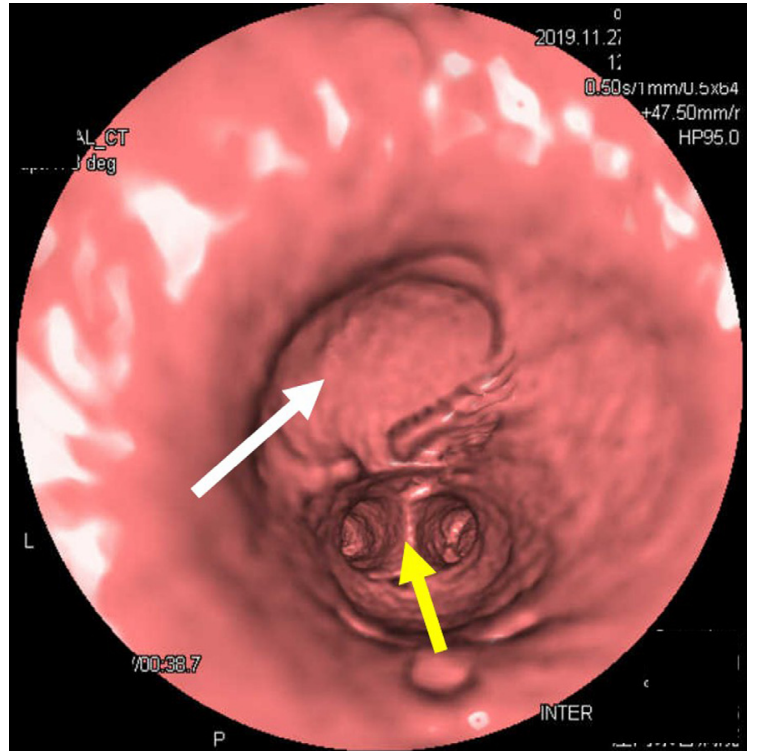

Figure 5 CT scan (intra-aortic view) of case 2 showing the entrance of the AAA from the proximal site. The white arrow points to the dome-shaped entrance of the saccular aneurysm. The yellow arrow points to a bifurcation of common iliac arteries.

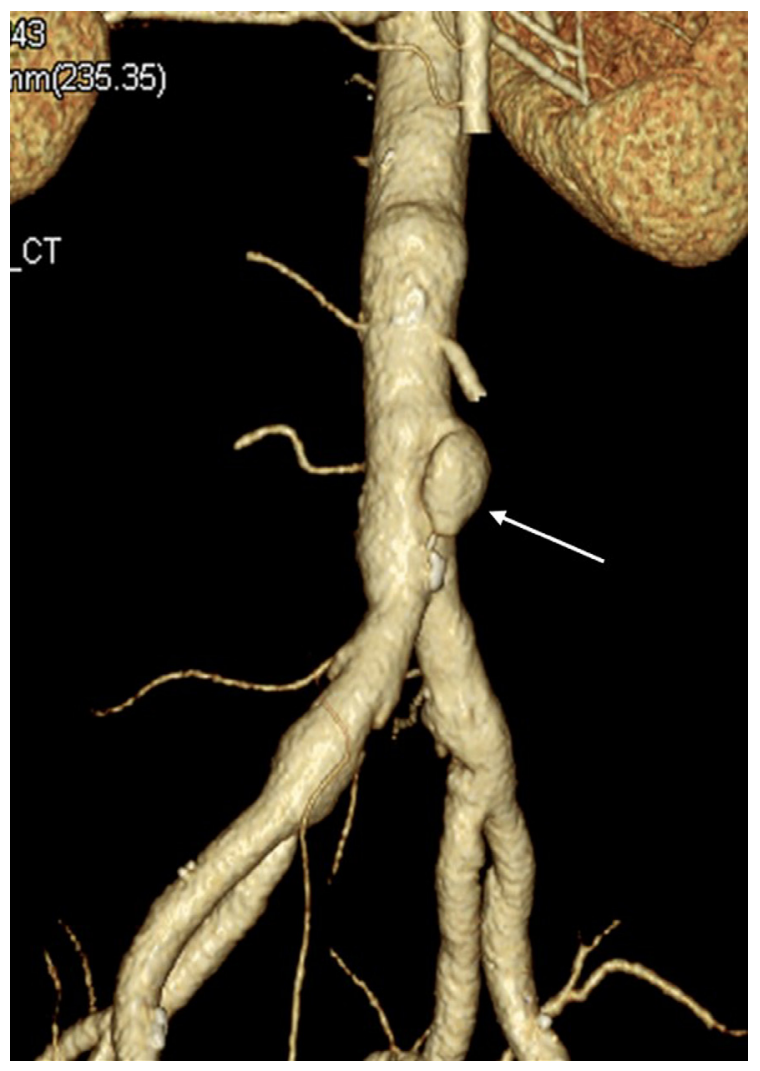

Figure 6 Enhanced three-dimensional CT scan of case 2. The white arrow points to the saccular aneurysm.

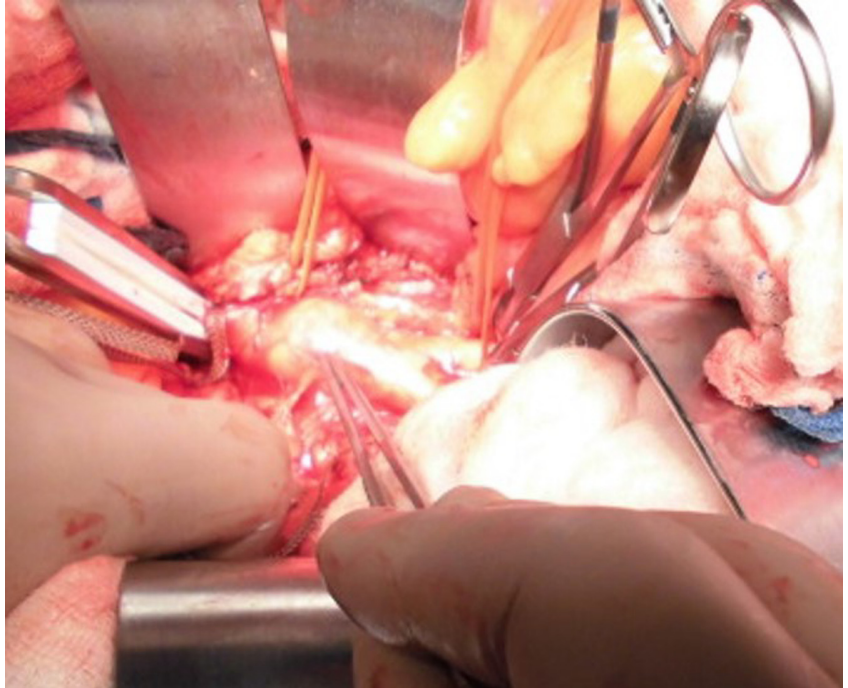

Figure 7 Intraoperative photograph. The wall of the saccular aneurysm was fragile, and a gentle push of the forceps penetrated the aneurysmal wall.

control, the shape and diameter of the AAA are important considerations. Although it is still controversial, a previous study strongly suggested that saccular aneurysms require immediate surgery regardless of the size ${ }^{6}$. Conversely, a recent review article ${ }^{2)}$ suggested that intervention should be considered when the diameter exceeds $30 \mathrm{~mm}$ because the minimum reported diameter of ruptured AAAs with IAAD is $35 \mathrm{~mm}^{7}$. Unfortunately, the number of cases reported in studies to date is insufficient to definitively determine the surgical indication of AAA with IAAD.

In case 1, EVAR was performed 3 years after the onset of IAAD. During the 3 years before EVAR, the patient had been asymptomatic; however, he complained of abdominal discomfort for the first time at 3 years after the onset of IAAD. At this time, the diameter of the saccular AAA already exceeded $30 \mathrm{~mm}$. We decided to perform surgery because of the abdominal symptoms and the AAA diameter. In case 2 , the AAA diameter was $<30 \mathrm{~mm}$; however, the patient complained of mild but continuous abdominal discomfort since onset. Although the AAA was still small, it was saccular. Most important, the patient was concerned about the risk of rupture and wished to undergo early surgery. We chose laparotomy and graft replacement rather than EVAR after a careful consideration of the patient's condition. We considered that the wall of the aneurysm in the subacute phase may be fragile and, consequently, easily susceptible to catheter penetration. Preoperative examination showed that the patient could endure the invasiveness of laparotomy. At the time of surgery, we carefully exposed the wall of the aneurysm (Figure 7), which we confirmed to be rather fragile. After clamping the proximal and distal sites of the AAA, a gentle push with forceps easily penetrated the AAA wall. 


\section{Journal of Rural Medicine}

In conclusion, the coexistence of IAAD and AAA is a rather dangerous condition; however, no established surgical indications are available thus far. Persistent pain or AAA diameter $>30 \mathrm{~mm}$ plays an important role in the decision making about surgery. At present, patients should be managed on a case-by-case basis. Accumulation of cases would contribute to the future development of treatment guidelines for AAA with IAAD.

Conflict of interest: The authors declare no conflicts of interest in association with the present study.

\section{References}

1. Jonker FH, Schlösser FJ, Moll FL, et al. Dissection of the abdominal aorta. Current evidence and implications for treatment strategies: a review and metaanalysis of 92 patients. J Endovasc Ther 2009; 16: 71-80. [Medline] [CrossRef]

2. Dodos IA, Theodoridis P, Staramos D, et al. Endovascular repair of isolated abdominal aortic dissection. Literature review. Hellenic Journal of Surgery 2018; 90: 85-89. [CrossRef]

3. Pereira AH. Intramural hematoma and penetrating atherosclerotic ulcers of the aorta: uncertainties and controversies. J Vasc Bras 2019; 18: e20180119e20180119. [Medline] [CrossRef]

4. Gifford SM, Duncan AA, Greiten LE, et al. The natural history and outcomes for thoracic and abdominal penetrating aortic ulcers. J Vasc Surg 2016; 63: 1182-1188. [Medline] [CrossRef]

5. Yoon SH, Maeno Y, Kawamori H, et al. Diagnosis and outcomes of transcatheter aortic valve implantation in bicuspid aortic valve stenosis. Interv Cardiol (Lond) 2018; 13: 62-65. [Medline]

6. Anderson CS, Heeley E, Huang Y, et al. INTERACT2 Investigators Rapid blood-pressure lowering in patients with acute intracerebral hemorrhage. N Engl J Med 2013; 368: 2355-2365. [Medline] [CrossRef]

7. Farber A, Lauterbach SR, Wagner WH, et al. Spontaneous infrarenal abdominal aortic dissection presenting as claudication: case report and review of the literature. Ann Vasc Surg 2004; 18: 4-10. [Medline] [CrossRef] 